\title{
The effects of antipsychotic drugs and symptomatology on perceptual asymmetry in schizophrenia
}

\author{
VAUGHAN CARR, JOCELYN WALE \\ From the Department of Psychiatry, University of Adelaide, and the Department of Psychiatry, Royal Adelaide \\ Hospital, Adelaide, South Australia.
}

SUMMARY Perceptual asymmetry was measured in acutely psychotic schizophrenic patients and normal controls using a dichotic monitoring task. Although the schizophrenic group differed from the controls in terms of accuracy and speed of response, there were no significant group differences with respect to asymmetries in these variables. The pattern of responding in the schizophrenic group was also examined in relation to differences in symptomatology and antipsychotic drug administration. These variables, in isolation and in interaction with each other, were found to have a number of effects on both asymmetry and overall level of responding. The results were interpreted in the context of an information processing model of functional hemisphere asymmetry in schizophrenia, and the implications regarding the nature of schizophrenic symptomatology and the effects of antipsychotic drugs were discussed.

The paradigm of asymmetry in cerebral hemisphere functioning has been widely used in studies of psychiatric disorders, especially schizophrenia. Although a number of differences between schizophrenic and control subjects have been reported, a consistent pattern of abnormality has not yet emerged which is capable of resolving the competing explanations of this disorder in terms of cerebral laterality. One important source of this failure may lie in the different experimental methods used to investigate this area. These have included electrodermal measurements, dichotic monitoring, electroencephalographic and evoked potential studies, neuropsychological testing, assessment of handedness, lateral eye movements and, more recently, regional cerebral metabolism and blood flow studies. This is not an exhaustive list but it highlights the wide diversity of experimental approaches employed within the functional hemisphere asymmetry paradigm.

Two other potential sources of variability include clinical heterogeneity and treatment effects, particularly those of antipsychotic drugs. With regard to

Address for reprint requests: Dr Vaughan Carr, Department of Psychiatry, Royal Adelaide Hospital, Adelaide, South Australia, 5000 .

Received 26 April 1988.

Accepted 1 July 1988 the former, symptomatology has emerged as an important parameter to be taken into account. Gruzelier $^{12}$ has reviewed this subject and found that, differences in methodology notwithstanding, left hemisphere activation tends to be associated with paranoid or reactive subtypes of schizophrenia and is linked with symptoms described as "hallucinatory, florid, and positive". In contrast, he found that right hemisphere activation coincided with catatonic, residual or non-paranoid subtypes and symptoms described as depressive, non-hallucinating, negative and withdrawn/retarded. ${ }^{2}$ Differences in performance asymmetry according to symptomatology have also been found on one dichotic monitoring task but not on another. ${ }^{3}$ These results were thought to reflect the different demands made on attention by each task. Thus the notion was raised of more specific asymmetries of cognitive functioning rather than overall hemispheric differences.

The issue of treatment effects is less clear by comparison. This may be partly due to the variations in what constitutes treatment in different cases, its duration and the relative contributions of antipsychotic drugs and other therapies. Each of these factors may further compound the problems arising from the various experimental paradigms mentioned above. Evoked potential studies in schizophrenia have demonstrated greater right-sided amplitude of late 
visual evoked potential (VEP) components in medicated patients with reversal of this asymmetry in drug-free patients. ${ }^{4}$ Furthermore, this drug-related enhancement of the late VEP component on the right side was reported to increase in proportion to antipsychotic drug doses. ${ }^{45}$ Other investigators have reported less alpha power in the EEG over the right hemisphere in schizophrenic patients prior to antipsychotic drug treatment and normalisation in this pattern of laterality after treatment. ${ }^{6}$ An important study of regional cerebral blood flow ( $\mathrm{CCBF}$ ) in schizophrenia by Gur $e t$ al demonstrated increased resting flow in the left hemisphere, especially in the more severely disturbed patients. This finding with respect to clinical profile appears to be consistent with the review of Gruzelier ${ }^{2}$ in which florid symptoms tended to be associated with left hemisphere activation. In addition to anomalies in rCBF with activation by certain cognitive tasks, Gur et al compared their results with a previous study of medicated patients and found that antipsychotic drugs appeared to restore symmetry of resting rCBF and that this occurred before symptomatic relief. ${ }^{7}$ Disappearance of the asymmetry in the Hoffman reflex with antipsychotic drug treatment has also been reported in schizophrenic patients. ${ }^{8}$

Dichotic listening studies have demonstrated changes in cerebral laterality with treatment. Wexler and Heninger ${ }^{9}$ reported an increase in the right ear advantage (REA) on a dichotic nonsense syllable test in depressed and schizophrenic subjects who had recovered from psychosis. In an extension of this work using a different dichotic task, the fused rhymed word test, Wexler ${ }^{10}$ reported a reduction in perceptual asymmetry with recovery in a diagnostically heterogeneous group of psychotic subjects. This unexpected change did not appear to be due to medication and, with the exception of a successful replication of the earlier results using the dichotic nonsense test, there was no change in perceptual asymmetry with the other dichotic tests used. Finally, Johnson and Crockett ${ }^{11}$ reported an absence of the normal REA on a dichotic words test in schizophrenia while the expected left ear advantage (LEA) on a dichotic chords test was obtained. Following treatment, the schizophrenic subjects showed a REA on the latter test and increased their performance on the right ear in the dichotic words test. These investigators interpreted their results to suggest that in schizophrenia there is a disruption of the interhemispheric inhibition mediating normal perceptual asymmetry and that treatment brings about an abnormal pattern of asymmetry reflecting the allocation of both verbal and nonverbal material to the left hemisphere. The merit of this conclusion, issues of validity aside, lies in its greater specificity than explanations offered simply in terms of over- versus underactivity of one hemisphere or another. The importance of carefully selecting tasks according to the cognitive processes they entail is also highlighted by the differences found between various dichotic tasks.

Some of the questions raised by these studies include the following. What are the differential effects of antipsychotic drugs and other therapeutic modalities on the various indices of functional cerebral asymmetry? Do the immediate effects of antipsychotic drugs on these indices differ from those of delayed effects? Is there a differential effect of these treatment variables depending on clinical characteristics such as symptom profile or diagnostic subtype? The present investigation attempted to examine the differential effects of recently administered antipsychotic drugs on dichotic monitoring indices of functional cerebral asymmetry according to variations in the relative preponderance of delusions, hallucinations and negative symptoms in schizophrenic patients. Previous research using the dichotic monitoring task has shown variations in the obtained ear differences according to symptomatology, ${ }^{3}$ but the possible effects of medication on these asymmetries is not known.

\section{Method}

\section{Subjects}

There were 26 subjects ( 19 males, 7 females) who met DSMIII criteria ${ }^{12}$ for schizophrenia $(\mathrm{N}=23)$ or schizophreniform disorder $(\mathrm{N}=3)$. The mean age was 25.4 years $(\mathrm{SD}, 6.8$; Range, 15-42). They were matched for age and sex with 26 normal controls. The schizophrenic and schizophreniform subjects were recently admitted acutely psychotic inpatients on short term treatment units. Diagnosis was based on case record review, clinical interview by a senior consultant psychiatrist (V.C.) and post-discharge follow-up over a period of 12 to 18 months. The 26 patients, hereafter referred to as the schizophrenic group, and the normal controls were assessed for handedness using the Annett handedness questionnaire. ${ }^{13}$ The distribution of hand preferences for each group indicated that the schizophrenic and normal control subjects did not differ significantly on this variable (Schizophrenia: right 19, left 4, mixed 3; Control: right 24, left 2, mixed 0 ). Of the schizophrenic subjects, 15 were free of antipsychotic drugs and 10 of these had been drug-free for more than 30 days. The 11 subjects who had been prescribed medication were taking an average of $\mathbf{4 4 8}$ chlorpromazine equivalents per day (SD, 345) with a mean treatment duration of $2 \cdot 3$ days (SD, 1.3). Thus, antipsychotic drug administration was in relatively low dosage over a very short period.

\section{Procedure}

The schizophrenic patients were interviewed and administered the Brief Psychiatric Rating Scale (BPRS) ${ }^{14}$ by one of the authors (V.C.) within 72 hours of hospital admission. A dichotic monitoring test was administered within this same time, often on the same day as the clinical assessment. The dichotic monitoring task has been described in detail elsewhere. ${ }^{\text {Is }}$ This test requires the subject to press a response button whenever a target word is heard. Lists of 120 word 
pairs containing 20 targets (10 for each ear), phonemic distractors and irrelevant words presented simultaneously, one to each ear, at a rate of $1 / 750 \mathrm{~ms}$. Responses and stimuli were recorded on an Apple IIe computer for further analysis as described by Wale and Geffen.$^{16}$ Stimuli for this task were presented using Maico auraldome headphones and a Uher two channel tape recorder. Programmes for the scoring and analysis of individual subject results were obtained from the laboratory where the test was developed.

The dichotic monitoring test was preceded by a brief test of hearing consisting of two tones which are heard centrally if hearing is equal in each ear, ${ }^{15}$ and a shaping procedure consisting of monaural lists. Since audiometric testing was not readily available, subjects were excluded if these preliminary procedures revealed any evidence to suggest reduced hearing in one ear compared to the other. After two dichotic practice lists, four tests were administered. The hand of response was counterbalanced in a $R / L / L / R$ order, and headphones were reversed after alternate lists. Two measures for each ear were derived from this procedure. These were the percentage of correct responses or target detection accuracy and the reaction time. Reaction times were not available for one schizophrenic subject owing to computer failure.

The symptoms of interest on the BPRS were delusions, hallucinations and negative symptoms. These were measured by combining certain BPRS items. Addition of items 11 (suspicousness) and 15 (unusual thought content) formed the delusion scale, item 12 (hallucinatory behaviour) comprised the hallucination scale, and combined scores on items 3 (emotional withdrawal), 13 (motor retardation) and 16 (blunted affect) made up the negative symptom scale. All schizophrenic subjects received scores on each of these symptom scales.

\section{Results}

The schizophrenic and control subjects were first compared in terms of target detection (TD) and reaction time (RT) using two repeated measure ANOVAs, group $(2) \times$ ear(2). With respect to TD, normal control subjects performed at a higher level (Left: $\overline{\mathrm{x}}=84.33 \%$, SD $=10 \cdot 62$; Right: $\overline{\mathrm{x}}=91.25 \%$. $\mathrm{SD}=6.61$ ) than did those with schizophrenia (Left: $\overline{\mathrm{x}}=63.46 \%, \quad \mathrm{SD}=19.55 ; \quad$ Right: $\overline{\mathrm{x}}=71.44 \%$, $\mathrm{SD}=16.08), \mathrm{F}(1,50)=36.00, \mathrm{p}<0.001$. Targets were responded to more often in the right ear $(\overline{\mathrm{x}}=81.35 \%, \mathrm{SD}=15.75)$ than those in the left $(\overline{\mathrm{x}}=73.89 \%, \mathrm{SD}=18.80), \mathrm{F}(1,50)=14.47, \mathrm{p}<$ 0.001 , but the interaction of group with ear was not significant. Thus ear asymmetries did not vary between the two groups. Similar main effects of group and ear were obtained on the RT measure. Responses for the control group (Left: $\overline{\mathrm{x}}=566 \mathrm{~ms}, \mathrm{SD}=82$; Right: $\bar{x}=535$, SD $=62$ ) were faster than those of the schizophrenic group (Left: $\bar{x}=685 \mathrm{~ms}$, $\mathrm{SD}=171 ; \quad$ Right: $\quad \overline{\mathrm{x}}=634, \quad \mathrm{SD}=96$ ), $F(1,49)=15.92, p<0.001$, and responses were faster to right ear stimuli $(\overline{\mathrm{x}}=583, \mathrm{SD}=94)$ than to left $(\overline{\mathrm{x}}=625, \mathrm{SD}=145), \mathrm{F}(1,49)=8.25, \mathrm{p}<0.01$. As with TD, the interaction of group with ear for RT was not significant. It was thought that the degree of variability in the data for the schizophrenic subjects, as reflected in the standard deviations, may be related to possible differences in performance asymmetry according to medication status and symptomatology. Medicated subjects did not differ from their unmedicated counterparts, however, in terms of symptom severity on any of the symptom dimensions in question (Mann-Whitney U-test).

In order to examine the possibility of differential effects of antipsychotic drugs on performance asymmetry depending on variations in symptomatology, subjects were divided into two groups depending in whether their symptom scores fell above or below the group median for a given symptom dimension. The aim was to examine the effect of drugs on the dichotic monitoring variables in relation to high versus low scores on each of the three symptom dimensions in turn. A series of planned comparisons was then performed on data for the schizophrenic group only in order to analyse possible effects associated with drug status and symptomatology. Repeated measure ANOVAs, symptom(2) $\times \operatorname{drug}(2) \times \operatorname{ear}(2)$, were undertaken using TD and RT separately as the dependent variables. These pairs of analyses were repeated for each of the three symptom dimensions.

Figure 1 shows the results for TD according to categorisation on the delusion scale and antipsychotic drug status. There were no main effects of ear, symptom rating or drug status. There was a significant two-way interaction between symptom rating and ear, $F=7.98(1,22), p=0.010$, with a high delusion score being associated with increased right ear performance. There was also a significant two-way interaction between drugs and ear, $F=4.75(1,22), p=0.040$, which reflected an association between antipsychotic medication and reduced right ear performance. There

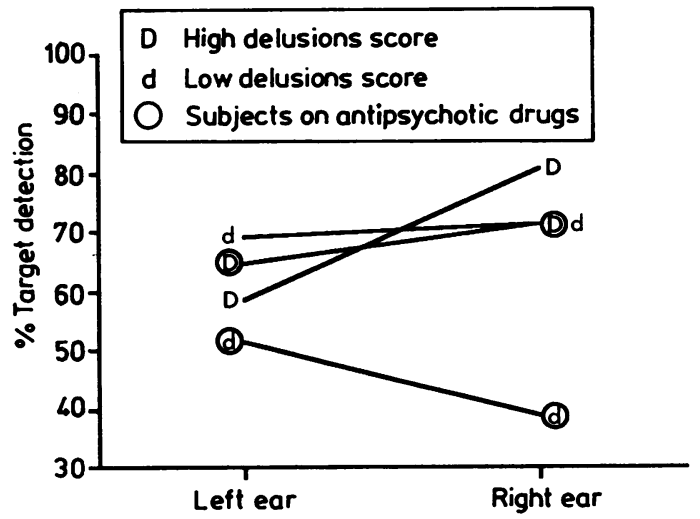

Fig 1 Results of repeated measure ANOVA comparing the effects of delusions and antipsychotic drugs on target detection for left and right ears. 


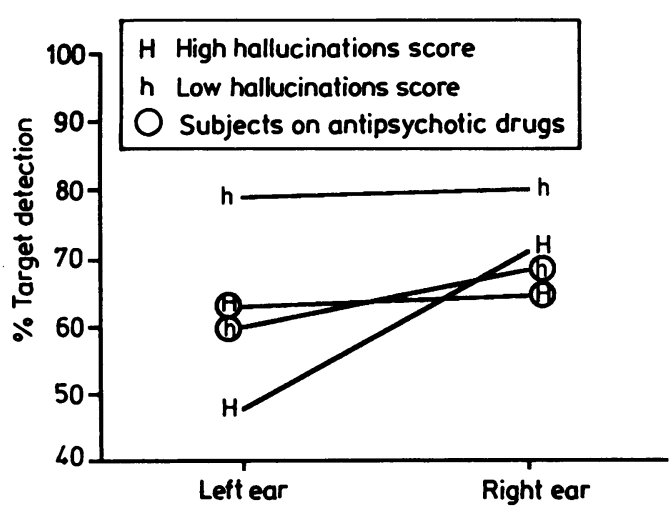

Fig 2 Results of repeated measure ANOVA comparing effects of hallucinations and antipsychotic drugs on target detection for left and right ears.

was no significant three-way interaction. The analysis of RT in relation to delusions and drug status revealed no significant main effects or interactions. Thus, the REA was found only in relation to a high rating for delusions or a drug-free status, and this occurred for level of correct performance but not response speed.

Figure 2 shows the results for TD according to categorisation on the hallucination scale and antipsychotic drug status. There was a significant main effect for ear, $F=7.24(1,22), p=0.013$, with right ear performance higher than left, but no significant main effects of symptom group or drug status. Neither was there a significant two-way interaction between symptom group and ear nor between drug status and ear. There was, however, a significant three-way (symptom $x$ drug $x$ ear) interaction, $F=5 \cdot 50(1,22), p=0.028$, in which a low level of left ear performance was found for drug-free subjects scoring high on the hallucination scale. Figure 2 also shows that drug-free subjects with few hallucinations have a higher level of performance on this variable, and there appears to be a relatively low performance asymmetry in this group as well. Post hoc comparisons (Student's $t$ test, onetailed) of left ear TD in the drug-free group revealed lower performance levels in subjects with high hallucination scores than those with low hallucination scores $(p<0.001)$. Similar analysis of subjects with high hallucination scores confirmed the impaired left ear performance in the drug-free subgroup compared to those on drugs $(p<0.05)$, whereas in the group with low hallucination scores the better left ear performance level in the medicated compared to the unmedicated subjects was significant at the $p<0 \cdot 10$ level only. The ANOVA performed with RT as the dependent variable revealed no significant main effects or interactions.

With respect to negative symptoms and TD, there were no main effects for symptom group or drug status, with that for ear just failing to reach statistical significance, $F=3.35(1,22), p=0.081$. There was a two-way interaction between symptom severity and drug status, $F=5.00(1,22), p=0.036$, in which a high negative symptom score and antipsychotic drugs together were associated with impaired TD performance (see fig 3a). There were no other significant twoway interactions and no three-way interaction was obtained. A post hoc comparison (Student's $t$ test, one-tailed) of right ear TD in subjects with high negative symptom scores revealed lower performance levels for the medicated subgroup than those who were unmedicated $(p<0.05)$. The same comparison using left ear TD just failed to reach statistical significance $(p<0 \cdot 10)$. Similar analyses of subjects on antipsychotic drugs confirmed the impaired performance in the subgroup with high negative symptom scores relative to those with few negative symptoms for both left ear $(p<0.05)$ and right ear $(p<0.005)$ scores.

In the ANOVA with RT as the dependent variable, the only significant main effect was that of ear, $F=4.93(1,22), p=0.038$, with right ear perfor-

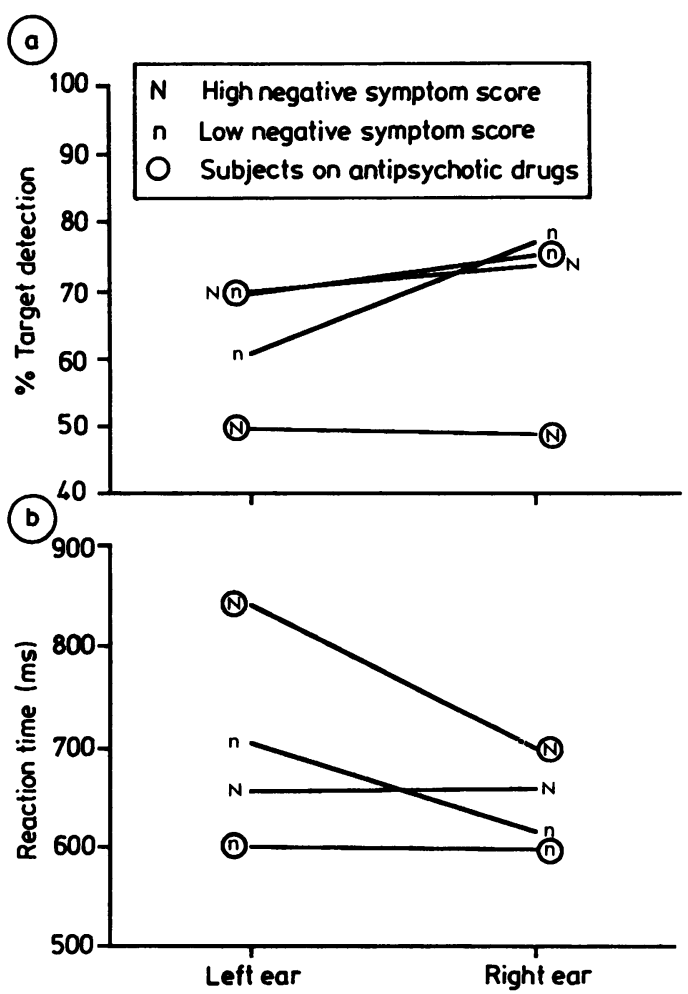

Fig 3 Results of repeated measure ANOVA comparing effects of negative symptoms and antipsychotic drugs on (a) target detection and $(b)$ reaction time for left and right ears. 
mance being faster than that of the left. The two-way interaction between symptom severity and drug status failed to reach statistical significance, $F=3 \cdot 25(1,22)$, $p=0.086$, but there was a significant three-way (symptom $x$ drug $x$ ear) interaction, $F=4.91(1,22)$, $p=0.038$, reflecting slower left ear performance for medicated subjects with high negative symptom ratings. These findings are illustrated in fig $3 \mathrm{~b}$. Post hoc comparisons using the RT measure failed to reach statistical significance.

Thus, subjects with relatively severe negative symptoms who are taking antipsychotic drugs show impaired performance on TD, and a tendency to poorer performance on RT ( $p<0 \cdot 10$ ), compared to drug-free subjects and those on drugs but with few negative symptoms. In terms of RT, medicated subjects with high negative symptom scores have an impaired left ear performance relative to the remaining subjects.

In summary, although perceptual asymmetry in schizophrenia did not differ from that found in ageand sex-matched normal control subjects, there were differences between these two groups in terms of accuracy of target detection and response speed. Secondly, within the schizophrenic group differential effects of symptomatology and antipsychotic drug status on perceptual asymmetry were obtained. The TD measure was generally more sensitive to these effects in relation to delusions and hallucinations. The RT measure was more sensitive to the effects on perceptual asymmetry of antipsychotic drugs in relation to negative symptoms while TD reflected an overall adverse effect on performance of medication in subjects scoring high on this symptom dimension. Thus, despite the rather small sample size and the associated low statistical power, significant effects were obtained which have some importance with respect to our understanding of schizophrenia in terms of perceptual asymmetries.

\section{Discussion}

Compared with normal controls, patients with acute schizophrenic psychosis show an impairment on the information processing task involved in the dichotic monitoring paradigm used in this study. This conclusion is drawn from the finding of reduced accuracy of target detection and prolonged reaction time in the schizophrenic group relative to the controls. This finding merely serves to add to the overwhelming amount of published evidence in support of a decrement in information processing in schizophrenia. However, in terms of both TD and RT, there is no evidence in the present study to support a global abnormality in functional hemisphere asymmetry in this disorder. This finding stands in contrast to those studies in which abnormalities in functional asymmetry have been reported. Nevertheless, as emphasised at the outset, the patterns of asymmetry reported elsewhere seem to vary depending on the nature of the experimental method used, certain clinical variables, especially symptomatology, and the effects of treatment. The mode of information processing, as governed by the nature of the cognitive task, appears from the literature reviewed to be particularly important in determining the patterns of asymmetry obtained. When symptomatology and short-term antipsychotic drug effects were addressed in the present study, which used a language processing task, a number of interesting findings emerged.

The results reported above suggest that delusions are associated with relative left hemisphere activation, a finding consistent with the review of Gruzelier. ${ }^{12}$ This finding implies an association between delusions and a relative augmentation of sequential processing operations which are predominantly left hemispherebased. The relationship between delusions and a relative increase in the accuracy with which verbal inputs presented directly to the language-dominant hemisphere are processed seems to be compatible with the notion that delusions represent a heightening of the normal processes involved in the ideational organisation of inputs. ${ }^{17}$ In this context, the association between antipsychotic drugs and a lower right ear performance level suggests that these drugs reduce left hemisphere activation and, hence, damp down the relative overactivation in sequential processing operations upon which delusions may be based. Although this may be the means by which antipsychotic drugs have their therapeutic effect in relation to delusions, the absence of a significant interaction between drug status and severity of delusions does not support a differential effect of antipsychotic drugs on functional hemisphere asymmetry according to variations in the severity of these symptoms. Rather, the therapeutic drug effect in this instance may be non-specific or indirect, reducing left hemisphere activation regardless of the extent of delusional ideation.

The situation with respect to hallucinations is more complex by comparison. In this case, there is a differential effect of antipsychotic drugs on functional hemisphere asymmetry according to the severity of these symptoms. Drug-free schizophrenic patients with high hallucination scores have a relatively impaired left ear performance on TD compared with both their medicated counterparts and those with lower hallucination scores. The net effect of such an impaired left ear performance in this group is to maintain a pattern of ear asymmetry in relation to hallucinations which would imply left hemisphere activation relative to right, consistent with the review of Gruzelier, ${ }^{12}$ except that the relative left ear perfor- 
mance decrement is here interpreted as evidence for an impaired ability of the left hemisphere to detect or attend to verbal inputs passing to it from the right hemisphere across the corpus callosum, rather than as evidence for right hemisphere inactivation relative to left. This interpretation is based on the nature of the dichotic monitoring task in this study where verbal stimuli are used, and therefore variations in TD need to be explained in terms of the functioning of the language-dominant hemisphere. On the other hand, drug-free schizophrenic subjects with low hallucination scores appear to have a superior overall level of performance compared to their medicated counterparts and those with relatively severe hallucinations. This result seems to highlight the potential for adverse effects on performance brought about by both the hallucinated state and medication. The pattern of the differential effects of antipsychotic drugs according to symptomatology suggests that drugs may be associated with an improvement in left ear performance (that is, left hemisphere attention to nondominant inputs) in those with relatively severe hallucinations but may actually impair this function in subjects with few hallucinations.

There were also some important differential effects of antipsychotic drugs on hemisphere asymmetry according to variations in the severity of negative symptoms. First, the combination of severe negative symptoms and antipsychotic drugs was associated with low performance levels bilaterally. In association with each other, these two variables thus appear to effect a reduction in left hemisphere processing of all verbal information, both dominant and non-dominant. Second, the results for RT suggest that there was also a differential interference with the speed with which the left hemisphere processed non-dominant (that is, left ear) inputs in medicated subjects with severe negative symptoms. Thus, in spite of a general decrement in information processing being associated with the administration of antipsychotic drugs to schizophrenic patients with high levels of negative symptoms, there is a further effect of interference with the efficiency of left hemisphere processing by these drugs in combination with negative symptoms.

In attempting to interpret these findings in relation to both the schizophrenic disorder itself and the short term effects of antipsychotic drugs, an information processing model of schizophrenia which provides for functional hemisphere asymmetries would appear to suit the data in this study. In an earlier publication ${ }^{17}$ we presented such a model in which a primary abnormality was proposed in a process referred to as preattentive grouping for which the right hemisphere is normally thought to be predominant. Compensatory mechanisms based on alterations in the left hemisphere-dominant functions of selective attention and/or sequential processing operations were proposed to arise secondarily and were held to be responsible for the formation of hallucinations, delusions and negative symptoms. The view that schizophrenia may primarily involve a preattentive/ right hemisphere abnormality with secondary left hemisphere dysfunction is consistent with much contemporary research in this field as summarised and interpreted recently by Cromwell. ${ }^{18}$

On the basis of this model, the results of the present study are consistent with an interpretation of the basis of delusion formation (viz., selective augmentation of the sequential processing operations of the left hemisphere) as overactivity in the process of ideational organisation of inputs in compensation for the input disorganisation occurring as a result of faulty preattentive (right hemisphere) mechanisms. Short term administration of antipsychotic drugs appears to counteract this processing mode, but this effect may be non-specific in relation to delusions which, instead, may decline more as a result of other therapeutic variables. If so, this could account for the clinical observation that delusions tend to subside at a slower rate than other psychotic symptoms and may require a period of "unlearning" or reality testing before their complete disappearance.

In contrast, hallucinations seem to be linked with selective inattention by the left hemisphere to inputs passing to it from what may be the primarily dysfunctional right hemisphere. ${ }^{1718}$ This selective inattention can be viewed as another compensatory mechanism by which the left hemisphere attempts to deal with disordered inputs. Medication appears to counter this inattention in those with severe hallucinations, suggesting a more direct therapeutic effect of antipsychotic drugs in relation to these symptoms compared to delusions. High levels of arousal may result in a restriction of selective or focussed attention..$^{19}$ It could be postulated that it is an arousal-related narrowing of focus or limiting of inputs for processing that not only accounts for the lower level of performance to nondominant items on dichotic monitoring but results in an hallucinatory interpretation of information. In reducing arousal, antipsychotic drugs may exert their therapeutic effect on hallucinations by causing attentional mechanisms to be less restricted, thus enabling non-dominant inputs to be processed more effectively by the left hemisphere. That this effect may be operating primarily at the level of the left hemisphere is supported by the reported association between this hemisphere and selective attention to type of information. ${ }^{20}$ By comparison, the alternative explanation that antipsychotic drugs have a directly beneficial effect on the faulty preattentive mechanisms seems less likely.

Negative symptoms, which were originally 
proposed to be a consequence of a compensatory mechanism referred to as restricted attention, ${ }^{17}$ were here associated with a global decrement in information processing and an additional impairment in the efficiency or speed with which the left hemisphere processed right hemisphere inputs, but only in association with antipsychotic drug administration. This suggests that the combination of negative symptoms and antipsychotic medication is general in its effects and produces a non-selective reduction in the information processing capacity or capacity utilisation of the left hemisphere.

The more important implications of this work are two-fold. First, the view of left hemisphere dysfunction in schizophrenia secondary to a right hemispherebased abnormality in preattentive mechanisms is extended to encompass the notion of several, symptom-related, left hemisphere processing strategies brought to bear, presumably in varying combinations, in an effort to handle disordered inputs from the primarily dysfunctional hemisphere. These are selective augmentation in sequential processing (delusions), selective inattention to non-dominant inputs (hallucinations), and non-selective reduction in processing capacity or capacity utilisation (negative symptoms in combination with antipsychotic drugs). Second, the differential effects of antipsychotic drugs on information processing according to variations in symptomatology, including the potential for such drugs in interaction with certain symptom dimensions to impair information processing, suggest that more discriminating choices need to be made in relation to the drug treatment of schizophrenia.

The authors are grateful to Dr G Geffen of the Department of Psychology at Flinders University for her help and support in the use of the dichotic monitoring task.

This research was supported by grant number 850266 from the National Health and Medical Research Council of Australia.

\section{References}

1 Gruzelier JH. A critical assessment and integration of lateral asymmetries in schizophrenia. In: Myslobodsky MS, ed. Hemisyndromes. Psychobiology, Neurology, Psychiatry. New York: Academic Press, 1983;265-326.

2 Gruzelier JH. Hemispheric imbalances in schizophrenia. Int J Psychophysiology 1984;1:227-40.

3 Wale J, Carr VJ. Dichotic listening asymmetries and psychotic symptoms in schizophrenia: A preliminary report. Psychiatry Res 1988;25:31-9.

4 Myslobodsky MS, Mintz M, Tomer R. Neuroleptic effects and the site of abnormality in schizophrenia. In: Myslobodsky MS, ed. Hemisyndromes. Psychobiology, Neurology, Psychiatry. New York, Academic Press, 1983:347-88.

5 Mintz M, Tomer R, Myslobodsky MS. Neurolepticinduced lateral asymmetry of visual evoked potentials in schizophrenia. Biol Psychiatry 1982;17:815-828.

6 Merrin EL, Fein G, Floyd TC, Yingling CD. EEG asymmetry in schizophrenic patients before and during neuroleptic treatment. Biol Psychiatry 1986;21: 455-464.

7 Gur RE, Gur RC, Skolnick BE, Caroff S, Obrist WD, Resnick S, Reivich M. Brain function in psychiatric disorders. III. Regional cerebral blood flow in unmedicated schizophrenics. Arch Gen Psychiatry 1985; 42:329-34.

8 Tan U, Gurgen F. Modulation of spinal motor asymmetry by neuroleptic medication of schizophrenic patients. Int J Neurosci 1986;30:165-72.

9 Wexler BE, Heninger GR. Alterations in cerebral laterality during acute psychotic illness. Arch Gen Psychiatry 1979;36:278-84.

10 Wexler BE. Alterations in cerebral laterality during acute psychotic illness. Br J Psychiatry 1986;149:202-9.

11 Johnson $O$, Crockett $D$. Changes in perceptual asymmetries with clinical improvement of depression and schizophrenia. J Abnorm Psychol 1982;91:45-54.

12 American Psychiatric Association. Diagnostic and Statistical Manual of Mental Disorders. Third Edition, Washington, D.C. 1980.

13 Annett M. A classification of hand preference by association analysis. Br J Psychol 1970;61:303-21.

14 Overall JE, Gorham DR. The brief psychiatric rating scale. Psychol Reports 1962;10:799-812.

15 Geffen G, Traub E, Stierman I. Language laterality assessed by unilateral ECT and dichotic monitoring. J Neurol Neurosurg Psychiatry 1978;41:354-60.

16 Wale J, Geffen G. Hemispheric specialization and attention: effects of complete and partial callosal section and hemispherectomy of dichotic monitoring. Neuropsychologia 1986;24:483-96.

17 Carr VJ, Wale J. Schizophrenia: An information processing model. Aust NZ Psychiatry 1986;20:136-55.

18 Cromwell RL. An argument concerning schizophrenia: The left hemisphere drains the swamp. In: Glass A, ed. Individual Differences in Hemispheric Specialisation. New York: Plenum 1987;349-56.

19 Kahneman D. Attention and Effort. Englewood Cliffs, New Jersey: Prentice-Hall, Inc. 1973.

20 Harter MR, Aine C, Schroeder C. Hemispheric differences in the neural processing of stimulus location and type: effects of selective attention on visual evoked potentials. Neuropsychologia 1982;20:421-38. 